\title{
Los trabajadores sociales en las diferentes esferas de la estructura social
}

\section{Rogelio Gómez García}

Dpto. Sociología y Trabajo Social, Facultad de Educación y Trabajo Social, Universidad de Valladolid

<rgomga@soc.uva.es>

Gizarte-langileek erabateko garrantzia izan dute espainiar Estatuan gizarte-zerbitzuak osatu eta hedatzean, eta baita ere ongizate Estatua osatzean. Gizarte-langileen enpleguan oinarriko eremua den Estatuko eremuaz gain, azken urteotan rol garrantzitsua garatu dute merkataritza eta harremanetako eremuek. Artikulu honetan saiatzen gara kalkulatzen zein probabilitate-maila duen profesional batek bere lan-ekimena garatu ahal izateko gizarte-egituraren eremu zehatz batean, norberaren eta lanpostuaren aldagaien arabera. Estatu-mailako eremuko erakunde batean lan egiteko probabilitatean eragin positiboa duten aldagaiak dira adina eta biztanleriaren alorra; merkataritza-alorrean, diru-sarbideen aldagaia; eta harremanetako alorrean, diru-sarbideen aldagaia eta guraso, lagun edo ahaideekin bizitzea.

\section{HITZ-GAKOAK:}

Gizarte Langintza, gizarte-egitura, eremuak, gizartelangileak, odds ratio.
Los trabajadores sociales han sido pieza clave en la construcción y expansión de los servicios sociales en el Estado español, y en la construcción del Estado de bienestar. Además de la esfera estatal, que es la principal empleadora de los trabajadores sociales, las esferas mercantil y relacional han desarrollado un importante papel en estos últimos años. En este artículo, nos proponemos estimar la probabilidad de que un profesional desarrolle su actividad laboral en una determinada esfera de la estructura social, en función de variables individuales y del puesto de trabajo. Las variables que tienen un efecto positivo sobre la probabilidad de trabajar en una entidad de la esfera estatal son la edad y el sector de población; en la esfera mercantil, la variable ingresos; y en la esfera relacional, las variables ingresos y convivir con sus padres, amigos o parientes.

\section{PALABRAS CLAVE:}

Trabajo social, estructura social, esferas, trabajadores sociales, odds ratio. 


\section{Introducción}

El trabajo social en España se profesionaliza vinculado al desarrollo del sistema de protección denominado bienestar social. Para una mayor precisión, tendríamos que vincularlo principalmente a la creación e institucionalización de uno de los pilares del sistema de protección social, como el sexto sistema después de la educación, la salud, la vivienda, los ingresos mínimos y la formación para el empleo (Khan y Kamerman, 1987).

A modo de resumen, y siguiendo las aportaciones de Monzón et al. (2004: 30-35), el modelo de bienestar social en España se materializa a través del denominado sistema público de servicios sociales, que se desarrolla sobre dos pilares fundamentales: a) el reconocimiento de una serie de libertades (de asociación, religiosa), y de derechos sociales reconocidos en la Constitución de 1978; y b) el establecimiento de la responsabilidad pública en la definición, asunción y desarrollo de un sistema básico de protección social.

Esto provoca la aparición de nuevos sistemas de gestión basados en la descentralización. Por un lado, la administración central transfiere parte de su responsabilidad a regiones y entes locales. Por otro, genera la cooperación institucionalizada de las entidades no lucrativas en la política social y un incremento de la presencia del mercado en las políticas sociales. Los sectores públicos se adelgazan y crean nuevas formas indirectas de intervención en política social, con la participación del mercado y del tercer sector. Se produce, en definitiva, un cambio en el que surgen sistemas mixtos de bienestar, más descentralizados, más privatizados y abiertos a la colaboración institucional con el sector voluntario (Izquieta et al. 2008: 117).

A partir de la implantación de esta red de servicios sociales por parte de las comunidades autónomas y de las administraciones locales, se produce un incremento de la presencia laboral de profesionales en organismos e instituciones públicas (ministerios, consejerías de las administraciones autonómicas, ayuntamientos); en menor medida, en grandes empresas privadas; $y$, más recientemente, en diversas asociaciones relacionadas con problemáticas sociales específicas o dedicadas al abordaje de la marginalidad social.

Los datos que aparecen en la Tabla 1, aunque sean imprecisos y de difícil comparación entre sí, nos permiten vislumbrar la evolución del colectivo en las diferentes esferas de la estructura social. Así, podemos observar que, desde la década de los ochenta, los trabajadores sociales están más vinculados laboralmente a la esfera estatal que a la mercantil y relacional.

Este artículo tiene como objetivo descubrir aquellas variables que están más relacionadas, en la práctica profesional de los trabajadores sociales, con las diferentes esferas de la estructura social. Recoge una síntesis de un estudio más extenso realizado por Torices (2010) acerca de la realidad profesional de los trabajadores sociales que tenía como objetivos, por un lado, caracterizar el perfil socioprofesional, en sus dimensiones sociodemográfica, formativa y laboral; y por otro, conocer las necesidades y dificultades percibidas por los trabajadores sociales en relación a la formación, la inserción laboral y el ejercicio profesional.

Tabla 1. Esferas donde desarrollan su labor los trabajadores sociales, según diferentes estudios sociológicos

\begin{tabular}{|c|c|c|c|c|}
\hline Estudio & Ámbito geográfico & Esfera estatal & Esfera mercantil & Esfera relacional \\
\hline Vázquez (1971) & España & $\begin{array}{c}\text { (Estatal) } \\
28 \% \\
\end{array}$ & $\begin{array}{c}\text { (Privada) } \\
34 \%\end{array}$ & $\begin{array}{c}\text { (Paraestatal + Iglesia) } \\
37 \% \\
\end{array}$ \\
\hline $\begin{array}{l}\text { Pardavila y Esnal } \\
(1985)\end{array}$ & España & $\begin{array}{c}\text { (Administración central + } \\
\text { autonómica + diputaciones + } \\
\text { ayuntamientos) } \\
54,8 \%\end{array}$ & $\begin{array}{l}\text { (Ejercicio libre de } \\
\text { la profesión) } \\
0,6 \%\end{array}$ & $\begin{array}{c}\text { (Entidades privadas sin ánimo de } \\
\text { lucro + asociaciones + partidos } \\
\text { políticos } / \text { sindicatos) } \\
24,0 \%\end{array}$ \\
\hline $\begin{array}{l}\text { Llovet y Usieto } \\
(1990)\end{array}$ & $\begin{array}{l}\text { Comunidad de } \\
\text { Madrid }\end{array}$ & $\begin{array}{c}\text { (Ayuntamiento + comunidad } \\
\text { autónoma + administración } \\
\text { nacional) } \\
70,7 \%\end{array}$ & $\begin{array}{c}\text { (Privada laica) } \\
\quad 4,5 \%\end{array}$ & $\begin{array}{c}\text { (Patronazgo público + Iglesia) } \\
14,8 \%\end{array}$ \\
\hline Banda et al. (1999) & Huelva & $\begin{array}{c}\text { (Administración pública) } \\
71,5 \%\end{array}$ & & $\begin{array}{c}\text { (Cáritas, Cruz Roja, otras entidades } \\
\text { de iniciativa social) } \\
38,6 \%\end{array}$ \\
\hline $\begin{array}{l}\text { Instituto Ágora } \\
(2001)\end{array}$ & $\begin{array}{c}\text { Madrid } \\
\text { Cataluña } \\
\text { Andalucía } \\
\text { País Vasco } \\
\text { C. Valenciana } \\
\end{array}$ & $\begin{array}{l}\text { (Administración pública, } \\
\text { autonómica y provincial) } \\
22,1 \%\end{array}$ & $\begin{array}{c}\text { (Empresa privada) } \\
17,8 \%\end{array}$ & $\begin{array}{c}\text { (ONG y empresas /organizaciones } \\
\text { sin ánimo de lucro) } \\
53,1 \%\end{array}$ \\
\hline Fernández (2002) & Navarra & $\begin{array}{c}\text { (Administración pública) } \\
74,03 \%\end{array}$ & $\begin{array}{c}\text { (Empresa privada) } \\
5,78 \% \\
\end{array}$ & $\begin{array}{c}\text { (Tercer sector) } \\
\mathbf{2 0 , 1 9 \%} \\
\end{array}$ \\
\hline Cuesta Ruiz (2007) & La Rioja & $\begin{array}{c}\text { (Sector público) } \\
54,0 \% \\
\end{array}$ & $\begin{array}{c}\text { (Sector privado) } \\
7,6 \% \\
\end{array}$ & $\begin{array}{c}\text { (Tercer sector) } \\
35,3 \% \\
\end{array}$ \\
\hline $\begin{array}{l}\text { Berasaluze y } \\
\text { Berrio-Otxoa (2008) }\end{array}$ & $\begin{array}{c}\text { Álava } \\
\text { Bizkaia } \\
\text { Gipuzkoa }\end{array}$ & $\begin{array}{c}\text { (Administración pública) } \\
67,2 \%\end{array}$ & $\begin{array}{c}\text { (Empresa privada) } \\
10,1 \%\end{array}$ & $\begin{array}{c}\text { (Tercer sector) } \\
22,6 \%\end{array}$ \\
\hline
\end{tabular}

Fuente: Elaboración propia. 
El artículo se divide en cuatro apartados. En el primero, se expone el concepto de trabajo social y se desarrolla la estructura social como categoría de análisis, según distintos autores. En el segundo, se aborda la metodología empleada, así como las variables consideradas. En el tercero, se presenta una síntesis de las principales características demográficas, económicas y laborales de los trabajadores sociales, y se estima la probabilidad de que un trabajador social desarrolle su activad laboral en las diferentes esferas de la estructura social en función de una serie de variables individuales y del puesto de trabajo. Finalmente, se extraen unas conclusiones en las que se resumen los aspectos más relevantes del estudio.

\section{Trabajo social y estructura social}

El Consejo de Europa ha reconocido la relevancia y el papel del trabajo social, poniéndola de manifiesto en el documento Recomendaciones REC (2001) del Comité de Ministros a los Países miembros sobre los trabajadores sociales. El texto destaca que el trabajo social promueve el bienestar de individuos, grupos y comunidades, facilita la cohesión social en periodos de cambio, y da apoyo y protección a los miembros más vulnerables de la comunidad, trabajando conjuntamente con los usuarios, instituciones y profesionales. En el caso de algunas personas, las situaciones de conflicto o cambio afectarán a su capacidad de autonomía y de autosuficiencia, y harán que necesiten ayuda y orientación. Otras necesitarán asistencia, apoyo y protección. Los trabajadores sociales dan respuesta a estas necesidades: realizan una contribución esencial a la promoción de la cohesión social, tanto a través de un trabajo preventivo como dando respuesta a los problemas sociales. El trabajo social es, por lo tanto, una inversión para el futuro bienestar de Europa.

En esta inicial ubicación conceptual del trabajo social, es necesario retomar una categoría de análisis como es la estructura social. Según Leach y Udy (1974: 592-593), la palabra ‘estructura', en su significado original, hace referencia a la construcción de edificios, pero ya en el siglo XVI se empleaba también para denotar las relaciones entre las partes de un todo. De hecho, era generalmente utilizada en este sentido en los estudios anatómicos que, por entonces, empezaban a florecer. El paso del término de la anatomía a la sociología, aunque se produjo varios siglos después, fue una consecuencia lógica del empleo de analogías orgánicas por los pensadores políticos. La idea de que el estudio de la estructura social debería constituir un objetivo de la sociología parece deberse a Spencer (1858 [1966], citado en Leach y Udy [1974: 593]), quien hace referencia a "las inducciones de la sociología: hechos generales, estructurales y funcionales reunidos mediante la investigación de las sociedades y de sus cambios”. El hecho de que Spencer asocie los términos "estructura' y 'función' indica que partía de una imagen orgánica. Esa imagen aparece de nuevo en la obra de Durkheim, fuente de las ideas de Radcliffe-Brown.
En un marco funcional y sistémico, cabe incluir la concepción de Parsons sobre la estructura social: "La estructura social es un sistema pautado de las relaciones sociales de los actores" (Parsons, 1967: 199). Ahora bien, el autor participa en ese sistema a través de sus 'roles', con lo que la estructura social hará referencia a la combinación de roles y puede analizarse en tres planos: el de los valores, el de las instituciones y el de las colectividades. En sentido estricto, la estructura social se refiere al "sistema de roles diferenciados, debiendo analizarse los tipos de que se compone, cómo están distribuidos dentro del sistema social y cómo están integrados entre sí” (Parsons, 1966: 128).

Levy siguió analizando el concepto de estructura social, relacionándolo con el concepto de pauta o de cómo se hace lo que se hace, en tanto que la función implicaría lo que se hace. Así, la estructura significa "una pauta: es decir, una uniformidad observable de acción u operación” (Levy, 1952: 46). Levy distingue, basándose en Merton, entre: “a) las eustructuras 0 estructuras en que la acción afectada en términos de éstas se resuelve en eufunciones; y b) las destructuras o estructuras en que la acción afectada en términos de éstas se resuelve en disfunciones” (Levy, 1952: 82).

En los anteriores enfoques, la estructura social guarda una estrecha vinculación con lo sistémico, de modo que la estructura es del sistema social. Tal vinculación no puede afirmarse en el caso de Merton, que "al contrario de Parsons, no pasa de la lista de propiedades analíticas de la estructura social a la reconstrucción de la estructura como estructura de un sistema total, porque considera que las combinaciones de ese tipo no deben implicar necesariamente una estructura global del sistema o postular la necesidad de una integración” (Barbano, 1969: 177). En efecto, la integración es tan sólo una de las 26 propiedades analíticas de los grupos, y no plantea exigencias universales, sino de grados. Merton habla también de estructura social para referirse a las relaciones sociales institucionales, en tanto que la estructura cultural guardaría relación preeminente con la organización de valores normativos del comportamiento. El desfase estructural entre ambas estructuras desemboca en anomía (Merton, 1970: cap. 4).

En un marco interaccionista-simbólico y presidido por las relaciones de poder, Mills y Gerth enfocan el concepto de estructura social a partir del rol y de las instituciones:

\footnotetext{
La sociedad como estructura social está compuesta por los roles como segmentos diversamente combinados en su esfera global institucional. Las instituciones forman una estructura social y los cambios de la estructura social determinan el curso principal de la historia humana (Mills y Gerth, 1953: 14).
}

Marx (1859, citado en Leach y Udy [1974: 593]) había escrito ya acerca de las relaciones de producción diciendo que constituían "la estructura (Struktur) 
económica, la base (Basis) real sobre la que se levanta la superestructura (Uberbau) jurídica y política y a la que corresponden las formas (Formen) de la conciencia social determinada". La metáfora empleada por Marx es la de un edificio, no un organismo, pero la noción de estructura no aparece claramente diferenciada de otros conceptos. En otras obras, encontramos referencias a sistemas políticos, jurídicos, religiosos y filosóficos, en contextos en que la palabra 'sistema' se emplea en el mismo sentido que 'estructura', 'superestructura' y 'forma' (Lefebvre, 1962, citado en Leach y Udy [1974: 593]).

De cara a nuestro análisis, hemos partido del concepto de estructura social de Adelantado, Noguera y Rambla:

El conjunto de los modos en que las prácticas de grupos e individuos están organizadas (instituciones) y relacionadas entre sí (procesos sociales), de manera que se crean unos ejes de desigualdad que configuran la identidad de esos individuos y grupos, así como los cursos posibles de la acción social (individual y colectiva) [Adelantado et al., 2000: 29].

Adelantado et al. distinguen entre, por un lado, los factores globales o 'institucionales', y por otro, las categorías o grupos en que se distribuye la población en su interior, atendiendo a las distintas desigualdades. Con ello, quedaría una concepción de la estructura social en 'tres dimensiones': esferas de acción, ejes de desigualdad y actores colectivos. Las desigualdades atraviesan los contextos institucionales ya dados (esferas), dando lugar a actores colectivos que reestructuran lo existente.

Como señala Adelantado et al. (2000: 30-39), el concepto de 'esferas' institucionales o de acción no ha encontrado demasiado eco en la sociología clásica y serán autores más recientes quienes avalen la necesidad de definir esta dimensión de la estructura social (Giddens, 1994; Bourdieu, 1995; Santos, 1994; y Habermas, 1987, citados en Adelantado et al., 2000), distinguiendo cuatro esferas:

- La esfera mercantil: es una esfera de producción e intercambio material que jerarquiza las relaciones sociales según la capacidad de vender o comprar mercancías, y que irradia un proceso general de mercantilización de las relaciones sociales clave para entender la política social.

- La esfera estatal: incluye el aparato institucional y administrativo público, así como el conjunto de las actuaciones generadas en este mismo marco. Su mecanismo principal de coordinación pasa por la capacidad de regulación por vía normativa, y los procesos de administración y gestión de recursos.

- La esfera relacional: es la esfera compuesta por las acciones sociales supraindividuales que canalizan intereses y necesidades de las personas, mediante distintos grupos sociales distri- buidos en asociaciones formales y en grupos comunitarios.

- La esfera doméstico-familiar y de parentesco: abarca las actividades que se realizan dentro de las unidades mínimas de co-residencia en las que se ejecuta el denominado trabajo doméstico, que consiste en la prestación de cuidados personales (trabajo reproductivo) al margen de cualquier remuneración monetaria. En esta esfera subyace claramente la lógica patriarcal.

A partir de esta perspectiva, el trabajo social debe tener en cuenta, para su acción profesional, las cuatro grandes esferas o espacios de la sociedad: mercantil (esfera de producción e intercambio material), estatal (aparato institucional y administrativo público), doméstico-familiar (unidades mínimas de co-residencia, y vínculos sanguíneos y legales) y relacional (subesfera asociativa y subesfera comunitaria).

\section{Metodología: datos y variables}

Los datos en los que se basa este artículo provienen de un estudio descriptivo de los trabajadores sociales realizado por Torices (2010) en la Escuela Universitaria de Trabajo Social de León el curso 2009/2010 para el trabajo de fin de carrera, bajo la dirección del autor de este artículo.

El Consejo General de Colegios Oficiales de Diplomados en Trabajo Social y Asistentes Sociales ofrece información sobre dirección postal, teléfono, correo electrónico, número de colegiados y página web -en caso de poseerla- para cada uno de los colegios oficiales. Utilizando esta información, se envió un cuestionario en línea a los/as presidentes/as de los 37 colegios oficiales existentes en España para su estudio/valoración. Si el colegio decidía participar, debía enviar un correo a todos sus colegiados, adjuntando el cuestionario y las instrucciones necesarias para responderlo, y reenviarlo a una cuenta que se habilitó para recibirlos y resolver cualquier duda. El número de colegios que finalmente participaron fue de 14 , y el total de cuestionarios devueltos correctamente, de 485, si bien al final quedó reducido a 387 , que eran los que ejercían como trabajadores sociales. La recogida de la información se realizó durante los meses de marzo a mayo de 2010.

Con esta muestra y la población inicial, el error muestral cometido es del 5,05\%. La ficha técnica de la investigación se recoge en la Tabla 2.

Las variables consideradas para este artículo han sido las siguientes:

- Variable dependiente. Como variable dependiente hemos utilizado las diferentes esferas de la estructura social en la que los trabajadores sociales desarrollan su actividad profesional: esfera estatal, mercantil y relacional. 
Tabla 2. Ficha técnica de la investigación

\begin{tabular}{l|l}
\hline $\begin{array}{l}\text { Ámbito } \\
\text { geográfico }\end{array}$ & Nacional \\
\hline Universo & $\begin{array}{l}\text { Trabajadores sociales colegiados ejercientes de } \\
\text { todos los colegios profesionales }\end{array}$ \\
\hline $\begin{array}{l}\text { Tamaño del } \\
\text { universo }\end{array}$ & 29.553 colegiados en 37 colegios profesionales \\
\hline $\begin{array}{l}\text { Modo de } \\
\text { aplicación }\end{array}$ & Encuesta en línea \\
\hline $\begin{array}{l}\text { Tamaño de la } \\
\text { muestra }\end{array}$ & $\begin{array}{l}\text { Cáceres, Castilla-La Mancha, Galicia, Granada, } \\
\text { Gipuzkoa, León, Las Palmas, Navarra, Sevilla, } \\
\text { Valladolid) }\end{array}$ \\
\hline $\begin{array}{l}\text { Error } \\
\begin{array}{l}\text { Periodo de } \\
\text { recogida de la } \\
\text { información }\end{array}\end{array}$ & De marzo a mayo de 2010 \\
\hline
\end{tabular}

Fuente: Consejo General del Trabajo Social (2009).

- Variables independientes. Como variables independientes se han utilizado dos conjuntos de variables:

- Un conjunto de variables personales: sexo, edad, estado civil, convivencia, ideología, religiosidad, antigüedad en la profesión y situación profesional.
- Se ha considerado también lo que se han llamado variables del puesto de trabajo: salario, modalidad de contrato, dedicación y sector de población.

\section{Análisis de datos}

4.1. Características demográficas, económicas y laborales de los trabajadores sociales encuestados

En relación con la situación general de los trabajadores sociales, se destacan los resultados de las variables sociológicas referentes a sexo, edad, estado civil, forma de convivencia, ideología, religiosidad y antigüedad como profesional. Asimismo, y entre las variables asociadas al puesto de trabajo, se indican salario, tipo de contrato, jornada y sector de población. En la Tabla 3, se exponen los resultados del análisis descriptivo de las variables de la muestra, centrando nuestra atención en las esferas estatal, mercantil y relacional, dado que es el espacio en el que desempeñan su labor los trabajadores sociales.

Tabla 3. Características sociodemográficas y profesionales de los trabajadores sociales que han contestado a la encuesta (\%) $[\mathrm{N}=485]$

\begin{tabular}{|c|c|c|c|c|c|c|}
\hline & Variable & Valores & Total & Esfera estatal & Esfera mercantil & Esfera relacional \\
\hline \multirow{21}{*}{ 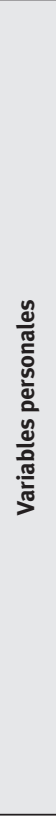 } & \multirow{2}{*}{ Situación } & Trabaja como trabajadora social & 79,8 & 58,4 & 11,7 & 29,9 \\
\hline & & Otras situaciones & 20,2 & - & - & - \\
\hline & \multirow{2}{*}{ Sexo } & Hombre & 9,7 & 72,2 & 2,8 & 25,0 \\
\hline & & Mujer & 90,3 & 56,6 & 12,9 & 30,5 \\
\hline & \multirow{3}{*}{ Edad } & $\leq 40$ años & 74,1 & 50,6 & 14,9 & 34,4 \\
\hline & & 41-50 años & 18,9 & 75,0 & 5,4 & 19,6 \\
\hline & & 51-61 años & 7,0 & 71,4 & - & 28,6 \\
\hline & \multirow{3}{*}{ Estado civil } & Soltera/o & 41,3 & 49,0 & 17,9 & 33,1 \\
\hline & & Casada/o o en pareja & 53,2 & 64,2 & 7,5 & 28,3 \\
\hline & & Separada/divorciada/viuda & 5,4 & 75,0 & 5,0 & 20,0 \\
\hline & \multirow{3}{*}{ Convivencia } & Con su marido/mujer / pareja & 64,9 & 62,1 & 9,5 & 28,4 \\
\hline & & Sola/o, con o sin hijos & 16,8 & 73,2 & 10,7 & 16,1 \\
\hline & & Con padres/amigos/parientes & 18,3 & 34,3 & 20,0 & 45,7 \\
\hline & \multirow{3}{*}{ Ideología } & Izquierda (1-4) & 72,2 & 57,1 & 13,1 & 29,8 \\
\hline & & Centro (5-7) & 24,3 & 58,8 & 10,6 & 30,6 \\
\hline & & Derecha (8-11) & 3,4 & 69,2 & - & 30,8 \\
\hline & \multirow{2}{*}{ Religiosidad } & Católico, creyente otra religión & 51,7 & 56,7 & 12,2 & 31,1 \\
\hline & & No creyente/ateo & 48,3 & 59,3 & 11,6 & 29,1 \\
\hline & \multirow{3}{*}{ Antigüedad } & $\leq 10$ años & 62,8 & 46,9 & 16,1 & 37,0 \\
\hline & & 11-20 años & 24,1 & 75,3 & 6,2 & 18,5 \\
\hline & & 21-38 años & 13,1 & 88,6 & - & 11,4 \\
\hline \multirow{9}{*}{ 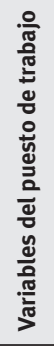 } & \multirow{3}{*}{ Ingresos } & $\leq 1.000 €$ & 14,5 & 16,7 & 25,9 & 57,4 \\
\hline & & $1.001-1.500 €$ & 40,8 & 44,0 & 16,0 & 40,0 \\
\hline & & $\geq 1.501 €$ & 44,7 & 86,8 & 2,6 & 10,5 \\
\hline & \multirow{2}{*}{$\begin{array}{l}\text { Tipo de } \\
\text { contrato }\end{array}$} & Indefinido & 72,0 & 62,2 & 11,6 & 26,3 \\
\hline & & De duración determinada & 28,0 & 50,0 & 12,3 & 37,7 \\
\hline & \multirow{2}{*}{ Jornada } & Jornada completa & 89,8 & 62,5 & 10,8 & 26,7 \\
\hline & & Jornada parcial & 10,2 & 28,9 & 15,8 & 55,3 \\
\hline & \multirow{2}{*}{$\begin{array}{l}\text { Sector de } \\
\text { población }\end{array}$} & Población en general & 20,7 & 91,9 & 4,1 & 4,1 \\
\hline & & Sectores de población & 79,3 & 49,6 & 13,7 & 36,6 \\
\hline
\end{tabular}

Fuente: Elaboración propia. 
Casi el $80 \%$ de los trabajadores sociales encuestados se encuentran ejerciendo la profesión, de los cuales el 58,4\% desarrollan su actividad profesional en la esfera estatal, el $29,9 \%$ en la relacional y el $11,7 \%$ restante en la mercantil.

Uno de los rasgos básicos de la profesión de trabajo social desde su origen sigue siendo la importante presencia de mujeres, el 90,3\% frente a un 9,7\% de hombres. Si observamos su distribución en las diferentes esferas en la que se encuentran desarrollando su actividad profesional, observamos cómo los hombres están principalmente en la esfera estatal $(72,2 \%)$, mientras que el porcentaje de mujeres en esta esfera se reduce al 56,6\%. La proporción de mujeres en la esfera mercantil asciende al 12,9\%, frente al 2,8\% de hombres.

Otro rasgo básico de la profesión es su juventud. Casi las tres cuartas partes $(74,1 \%)$ de los profesionales tienen menos de 40 años, y de éstos, el 50,6\% desarrollan su actividad en la esfera estatal, el 34,4\% en la esfera relacional y el 14,9\% restante en la esfera mercantil. Los profesionales de más de 40 años se encuentran principalmente en la esfera estatal: el $75 \%$ en el caso de las profesionales de 41 a 50 años y el $71,4 \%$ en el caso de los profesionales de más de 50 años.

En relación al estado civil de los trabajadores sociales encuestados, comprobamos que el 53,2 \% están casados o viven en pareja, mientras que los solteros representan el $41,3 \%$ y los separados/divorciados y viudos el 5,4\%. Si atendemos a su distribución en las diferentes esferas, obtenemos algunos datos más precisos. Los trabajadores sociales que están casados o viven en pareja y aquellos que están separados, divorciados o viudos desarrollan su actividad principalmente en la esfera estatal $(64,2 \%$ y $75 \%$, respectivamente), mientras que entre los solteros esta proporción se reduce hasta el $49 \%$.

Los trabajadores sociales encuestados conviven principalmente con su cónyuge o pareja en un $64,9 \%$ de los casos, mientras que el $18,3 \%$ de ellos lo hacen con sus padres, amigos o parientes, y un $16,8 \%$ vive solo (con o sin hijos). Tanto los que conviven con su cónyuge 0 pareja como los que viven solos (con o sin hijos) desarrollan su actividad principalmente en la esfera estatal $(62,1 \%$ y $73,2 \%$, respectivamente), mientras que los que conviven con sus padres, amigos o parientes trabajan principalmente en la esfera relacional (45,7\%).

El 72,2\% de los trabajadores sociales encuestados se sitúan en posiciones ideológicas de izquierda, un $24,3 \%$ en posiciones de centro y el 3,4\% restante en posiciones de derecha. Observando las diferentes esferas, vemos que en las tres posiciones ideológicas predominan los que desarrollan su actividad en la esfera estatal, aunque la concentración en dicha esfera entre quienes se sitúan en posiciones de derecha $(69,2 \%)$.
En términos de práctica religiosa, observamos porcentajes similares entre los que son católicos o creyentes de otra religión (51,7\%), y aquellos que no son creyentes o son ateos (48,3\%). Apenas hay diferencias según la esfera donde desarrollan su actividad.

Al analizar la antigüedad en la profesión, observamos que el $62,8 \%$ de los profesionales llevan menos de 10 años como tales; el 24,1\%, de 11 a 20 años; y el $13,1 \%$, de 21 a 38 años. Por esferas, la edad aparece directamente relacionada con la esfera estatal e inversamente relacionada con las esferas mercantil y relacional.

El 44,7\% de los trabajadores sociales encuestados tienen unos ingresos superiores a 1.500 euros; el 40,8\%, de 1.001 a 1.500 euros; y finalmente el $14,5 \%$ tiene ingresos inferiores a 1.000 euros. Los que se encuentran en la franja de ingresos superiores a 1.500 euros se concentran principalmente en la esfera estatal (86,8\%), la franja de 1.001 a 1.500 euros estaría repartida proporcionalmente entre la esfera estatal (44\%) y la esfera relacional (40\%), y finalmente los que ingresan menos de 1.000 euros se situarían principalmente en las esferas relacional (57,4\%) y mercantil $(25,9 \%)$.

Al analizar el tipo de relación laboral establecido, podemos ver que el $72 \%$ de los trabajadores sociales encuestados tienen una relación contractual de duración indefinida, frente a un $28 \%$ que tienen contrato temporal. Mientras que los que tienen una relación laboral de duración indefinida desarrollan su actividad principalmente en la esfera estatal $(62,2 \%)$, la presencia en esa esfera de los que tienen una relación laboral temporal se reduce hasta el $50 \%$.

Los trabajadores sociales encuestados, en un $89,8 \%$ de los casos, ejercen su actividad profesional con una dedicación completa, y en un 10,2\% de los casos, de forma parcial. Al observar su distribución entre las diferentes esferas, vemos cómo el 62,5\% de los que tienen una dedicación completa desarrollan su actividad en la esfera estatal, mientras que los que ejercen de forma parcial lo desarrollan en la esfera relacional $(55,3 \%)$.

Finalmente y tomando como referencia los sectores de población objeto de intervención, el 79,3\% de los trabajadores sociales encuestados atenderían a alguno de los diferentes sectores de población existente, mientras que el $\mathbf{2 0 , 7} \%$ se centrarían en la población en general. Entre los que tienen por objeto de intervención los diferentes sectores de población, observamos que el 49,6\% se encuentran en la esfera estatal y el $36,6 \%$ en la esfera relacional. Aquellos que atienden a la población en general se sitúan principalmente en la esfera estatal (91,9\%). 
4.2. Variables relacionadas con las diferentes esferas de la estructura social

La posible influencia de las distintas variables personales del individuo y del puesto del trabajo u organización va a efectuarse a través de un análisis preliminar, utilizando el indicador de correlación lineal de Pearson. Posteriormente, para cuantificar el grado de asociación entre las variables, se ha utilizado la odds ratio y su intervalo de confianza del $95 \%$.

La Tabla 4 recoge los resultados de la prueba chi cuadrado de Pearson para cada una de las variables que van a analizarse.

Tabla 4. Variables de los trabajadores sociales relacionadas con las diferentes esferas de la estructura social. Matriz de correlación lineal (coeficiente de Pearson)

\begin{tabular}{|c|c|c|c|c|}
\hline & \multirow[b]{2}{*}{$\begin{array}{c}\text { Esfera } \\
\text { estatal }\end{array}$} & \multirow[b]{2}{*}{$\begin{array}{c}\text { Esfera } \\
\text { mercantil }\end{array}$} & \multirow[b]{2}{*}{$\begin{array}{c}\text { Esfera } \\
\text { relacional }\end{array}$} \\
\hline & & & & \\
\hline \multirow{5}{*}{$\begin{array}{l}\text { Variables } \\
\text { personales }\end{array}$} & Sexo &, $107^{\star}$ & - & - \\
\hline & Edad &, $140^{\star \star}$ &,$- 151^{\star \star}$ &,$- 124^{*}$ \\
\hline & Estado civil &, $143^{\star \star}$ &,$- 156^{\star \star}$ & - \\
\hline & Convivencia &,$- 151^{\star \star}$ &, $122^{*}$ &, $116^{\star}$ \\
\hline & Antigüedad &, $329^{\star \star}$ & 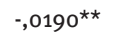 &,$- 222^{\star \star}$ \\
\hline \multirow{4}{*}{$\begin{array}{l}\text { Variables } \\
\text { del puesto } \\
\text { de trabajo }\end{array}$} & Ingresos &, $446^{\star \star}$ &,$- 267^{\star \star}$ &,$- 387^{\star \star}$ \\
\hline & Contrato & - & - &, $134^{\star \star}$ \\
\hline & Dedicación &,$- 178^{\star \star}$ & - &, $199^{\star \star}$ \\
\hline & Sector &,$- 347^{\star \star}$ &, $122 *$ &, $288^{\star \star}$ \\
\hline
\end{tabular}

* La correlación es significativa al nivel 0,05 (bilateral).

** La correlación es significativa al nivel o,01 (bilateral). Fuente: Elaboración propia.

Por lo que se refiere a los resultados obtenidos en el modelado de regresión logística binaria, la variable dependiente es la estructura social, la cual la hemos transformado en tres variables dicotómicas: esfera estatal ( 1 = sí, o = no), esfera relacional ( 1 = sí, $0=$ no) y esfera mercantil $(1=$ sí, $0=$ no).

\section{Las variables predeterminadas y su codificación son:}

- Sexo, considerada como dicotómica (mujer: ' 1 ' si lo es; ‘o’ si no lo es).

- Edad, considerada como factor con tres valores: menos de 40 años (que actúa como categoría base), de 41 a 50 años y de 51 a 61 años.

- Estado civil, considerada como factor con tres valores: casada/o o en pareja (que actúa como categoría base), soltera/o y separada/o-divorciada/o-viuda/o.

- Convivencia, considerada como factor con tres valores: con su marido-mujer-pareja (que actúa como categoría base), sola/o con o sin hijos, y con sus padres-amigos-parientes.

- Ideología, considerada como factor con tres valores: izquierda (que actúa como categoría base), centro y derecha.

- Religiosidad, considerada como factor con dos valores: no creyente-ateo (que actúa como categoría base) y católico-creyente de otra religión.

- Antigüedad, considerada como factor con tres valores: menos de 10 años (que actúa como categoría base), de 11 a 20 años y de 21 a 38 años.

- Ingresos, considerada como factor con tres valores: de 1.501 en adelante (que actúa como categoría base), de 1.001 a 1.500 euros, y hasta 1.000 euros.

- Tipo de contrato, considerada como dicotomizada (duración determinada: ‘1' si lo es, ‘o' si no lo es).

- Sector de población, considerada como dicotomizada (población en general: ' 1 ' si lo es, 'o' si no lo es).

El propósito es estimar la probabilidad de que un profesional desarrolle su actividad laboral en las diferentes esferas de la estructura social, en función de una serie de variables individuales y del puesto de trabajo. Lo que se persigue es construir un modelo que explique la mayor cantidad posible de variabilidad de la variable dependiente con el menor número posible de variables independientes (es decir, el modelo más parsimonioso).

\subsubsection{Variables relacionadas con la esfera estatal}

Las variables que se han incorporado como independientes son: sexo, edad, estado civil, convivencia, antigüedad, ingresos, jornada y sector de población. Entre todas las variables, se han incluido estas ocho porque son las que están correlacionadas con la esfera estatal.

Como puede verse en la Tabla 5, cuando el encuestado convive con los padres, amigos o parientes, la razón ${ }^{1}$ de trabajar en un centro de la esfera estatal disminuye 0,39 veces con respecto a la de los que conviven con su marido, mujer o pareja. Por el contrario, la antigüedad incrementa la razón de trabajar en la esfera estatal 3,01 veces en el caso de encuestados de 11 a 20 años de antigüedad y 12,63 veces para los que tienen de 21 a 38 años de antigüedad.

${ }^{1}$ La razón representa la frecuencia de ocurrencia de un suceso sobre la frecuencia de su no ocurrencia. En la interpretación de los resultados de un logit a través de los cocientes de razones, hay que tener muy en cuenta que no se está tratando directamente sobre las probabilidades de ocurrencia del suceso estudiado, sino sobre cómo varían las razones de ocurrencia del suceso en función de las variables independientes. 
Tabla 5. Odds ratios ajustados de desarrollar su labor en la esfera estatal, en función de diversas variables relacionadas con la persona y las condiciones laborales

\begin{tabular}{|c|c|c|c|c|c|c|c|}
\hline Variables & Especificación de las variables* & Odds ratio & Error estándar & $\mathbf{z}$ & $P>|z|$ & \multicolumn{2}{|c|}{$\begin{array}{l}\text { Intervalo de confianza del } \\
\qquad 95 \%\end{array}$} \\
\hline \multirow{2}{*}{ Sexo } & \multicolumn{7}{|l|}{ (Mujer) } \\
\hline & Varón & 2,045388 & ,9607626 & 1,52 & 0,128 &, 8146069 & 5,135743 \\
\hline \multirow{3}{*}{ Edad } & \multicolumn{7}{|l|}{ ( $\leq 40$ años $)$} \\
\hline & 41-50 años &, 5399282 &, 2186814 & $-1,52$ & 0,128 &, 2441085 & 1,194233 \\
\hline & 51-61 años &, 3645685 &, 2586201 & $-1,42$ & 0,155 & 0907711 & 1,464234 \\
\hline \multirow{3}{*}{ Estado civil } & \multicolumn{7}{|l|}{ (Casada/a o en pareja) } \\
\hline & Soltera/a & 1,389229 &, 5475592 & 0,83 & 0,404 & ,6416176 & 3,007957 \\
\hline & Separada/divorciada/viuda & 2,248127 & 1,869611 & 0,97 & 0,330 & ,4404896 & 11,47377 \\
\hline \multirow{3}{*}{ Convivencia } & \multicolumn{7}{|l|}{ (Con su marido/mujer/pareja) } \\
\hline & Sola/o, con o sin hijos & ,6862962 &, 3326776 & $-0,78$ & 0,437 & ,2653977 & 1,774704 \\
\hline & Padres/amigos/parientes &, 3945873 & ,1899377 & $-1,93$ & 0,053 & ,1536069 & 1,013621 \\
\hline \multirow{3}{*}{ Antiguiedad } & \multicolumn{7}{|l|}{$(\leq 10$ años) } \\
\hline & 11-20 años & 3,010118 & 1,060566 & 3,13 & 0,002 & 1,508956 & 6,004687 \\
\hline & 21-38 años & 12,63966 & 8,291776 & 3,87 & 0,000 & 3,494111 & 45,72295 \\
\hline \multirow{3}{*}{ Ingresos } & \multicolumn{7}{|l|}{$(\geq 1.501 €)$} \\
\hline & $\leq 1.000 €$ & ,0947059 & ,0470296 & $-4,75$ & 0,000 & ,0357834 &, 2506523 \\
\hline & $1.001-1.500 €$ & ,2329071 & ,0684473 & $-4,96$ & 0,000 & ,1309271 & ,4143201 \\
\hline \multirow{2}{*}{ Jornada } & \multicolumn{7}{|l|}{ (Jornada completa) } \\
\hline & Jornada parcial & ,8733997 & ,4471155 & $-0,26$ & 0,791 & ,3202295 & 2,382126 \\
\hline \multirow{2}{*}{$\begin{array}{l}\text { Sector de } \\
\text { población }\end{array}$} & \multicolumn{7}{|l|}{ (Sectores población) } \\
\hline & Población en general & 18,25585 & 8,943116 & 5,93 & 0,000 & 6,989053 & 47,68545 \\
\hline
\end{tabular}

\begin{tabular}{l|l}
\hline Number of obs & 382 \\
\hline LR chi $^{2}(19)$ & 174,42 \\
\hline Prob $>$ chi $^{2}$ & 0,0000 \\
\hline Pseudo $\boldsymbol{R}^{2}$ & 0,3308 \\
\hline Log likelihood & $-176,39485$ \\
\hline
\end{tabular}

* El valor de referencia se señala entre paréntesis.

Fuente: Elaboración propia.

En el caso de los ingresos, encontramos que cuando éstos se encuentran en el tramo de hasta 1.000 euros y en el de 1.001 a 1.500 euros, la razón de trabajar en la esfera estatal disminuye 0,09 veces y 0,23 veces, respectivamente, con respecto a los que cobran de 1.501 euros en adelante. Finalmente, observamos que para los que desarrollan su labor con la población en general, la razón de trabajar en la esfera estatal es 18,25 veces superior con respecto a los que lo hacen con diferentes sectores de la población.

Las variables que tienen un efecto positivo sobre la probabilidad de trabajar en la esfera estatal son: antigüedad (más probabilidad cuantos más años) y sector de población (población en general). Las variables convivencia e ingresos afectan negativamente. Por último, debemos señalar que la variable que tiene más efecto sobre la probabilidad de trabajar en una entidad de la esfera estatal es el sector de población (población en general).

\subsubsection{Variables relacionadas con la esfera mercantil}

Las variables que se han incorporado como independientes son: edad, estado civil, convivencia, antigüedad, ingresos y sector de población. Entre todas las variables, se han incluido estas seis porque son las que están correlacionadas con trabajar en una entidad de la esfera mercantil.

Como se puede observar, en este caso la variable ingresos incrementa la razón de trabajar en una entidad de la esfera mercantil 8,42 veces en el caso de tener unos ingresos de hasta 1.000 euros y 5,61 veces en el caso de tener unos ingresos de $1.001 \mathrm{a}$ 1.500 euros respecto al grupo de referencia (de 1.501 euros en adelante).

Desarrollar su actividad con la población en general disminuye en 0,33 veces la razón de trabajar en entidades de la esfera mercantil. La variable que tiene un efecto positivo sobre la probabilidad de trabajar en una entidad de la esfera mercantil es ingresos (más probabilidad cuantos menos ingresos). La variable sector de población (población en general) afecta negativamente. Por su parte, la variable que tiene más efecto sobre la probabilidad de trabajar en una entidad de la esfera mercantil es ingresos.

\subsubsection{Variables relacionadas con la esfera relacional}

Las variables que se han incorporado como independientes son: edad, convivencia, antigüedad, ingresos, tipo de contrato, jornada y sector de población. 
Tabla 6. Odds ratios ajustados de desarrollar su labor en la esfera mercantil, en función de diversas variables relacionadas con la persona y las condiciones laborales

\begin{tabular}{|c|c|c|c|c|c|c|c|}
\hline Variables & Especificación de las variables* & Odds ratio & Error estándar & $\mathbf{z}$ & $P>|z|$ & \multicolumn{2}{|c|}{$\begin{array}{c}\text { Intervalo de confianza } \\
\text { del } 95 \%\end{array}$} \\
\hline \multirow{2}{*}{ Edad } & \multicolumn{7}{|l|}{ ( $\leq 40$ años) } \\
\hline & 41-50 años & ,8550923 &, 5904414 & $-0,23$ & 0,821 & ,2209318 & 3,309542 \\
\hline \multirow{3}{*}{ Estado civil } & \multicolumn{7}{|l|}{ (Casada/o o en pareja) } \\
\hline & Soltera/o & 1,729441 & ,8444308 & 1,12 & 0,262 & ,6641882 & 4,50319 \\
\hline & Separada/divorciada/viuda & ,7313495 & ,9120446 & $-0,25$ & 0,802 &, 063477 & 8,426235 \\
\hline \multirow{3}{*}{ Convivencia } & \multicolumn{7}{|l|}{ (Con su marido/mujer/pareja) } \\
\hline & Sola/o, con o sin hijos & 1,189501 & ,7550959 & 0,27 & 0,785 & ,3427849 & 4,127699 \\
\hline & Padres/amigos/parientes & 1,114491 &, 5493002 & 0,22 & 0,826 & ,4241738 & 2,928256 \\
\hline \multirow{2}{*}{ Antigüedad } & \multicolumn{7}{|l|}{ ( $\leq 10$ años) } \\
\hline & 11-20 años & ,8198176 & ,4596397 & $-0,35$ & 0,723 & ,2732007 & 2,4601 \\
\hline \multirow{3}{*}{ Ingresos } & \multicolumn{7}{|l|}{$(\geq 1.501 €)$} \\
\hline & $\leq 1.000 €$ & 8,427014 & 5,278296 & 3,40 & 0,001 & 2,468994 & 28,76255 \\
\hline & $1.001-1.500 €$ & 5,614147 & 3,191334 & 3,04 & 0,002 & 1,842563 & 17,10587 \\
\hline \multirow{2}{*}{$\begin{array}{l}\text { Sector de } \\
\text { población }\end{array}$} & \multicolumn{7}{|l|}{ (Sectores población) } \\
\hline & Población en general & ,3327844 & ,2107073 & $-1,74$ & 0,082 & ,0962082 & 1,151102 \\
\hline
\end{tabular}

\begin{tabular}{l|l}
\hline Number of obs & 334 \\
\hline LR chi $^{2}(19)$ & 31,90 \\
\hline Prob chi $^{2}$ & 0,0002 \\
\hline Pseudo $\boldsymbol{R}^{2}$ & 0,1262 \\
\hline Log likelihood & $-110,3781$ \\
\hline
\end{tabular}

* El valor de referencia se señala entre paréntesis.

Fuente: Elaboración propia.

Tabla 7. Odds ratios ajustados de desarrollar su labor en la esfera relacional, en función de diversas variables relacionadas con la persona y las condiciones laborales

\begin{tabular}{|c|c|c|c|c|c|c|c|}
\hline Variables & Especificación de las variables* & Odds ratio & Error estándar & $\mathbf{z}$ & $P>|z|$ & \multicolumn{2}{|c|}{$\begin{array}{c}\text { Intervalo de confianza } \\
\text { del } 95 \%\end{array}$} \\
\hline \multirow{3}{*}{ Edad } & \multicolumn{7}{|l|}{ ( $\leq 40$ años) } \\
\hline & 41-50 años & ,9555036 &, 4176628 & $-0,10$ & 0,917 &, 4056592 & 2,250626 \\
\hline & 51-61 años & 1,832939 & 1,199984 & 0,93 & 0,355 &, 5080257 & 6,613182 \\
\hline \multirow{3}{*}{ Convivencia } & \multicolumn{7}{|l|}{ (Con su marido/mujer/pareja) } \\
\hline & Sola/o, con o sin hijos &, 5131192 & ,2191861 & $-1,56$ & 0,118 & ,2221342 & 1,18528 \\
\hline & Padres/amigos/parientes & 1,903852 & ,6390214 & 1,92 & 0,055 & ,9861213 & 3,675666 \\
\hline \multirow{3}{*}{ Antigüedad } & \multicolumn{7}{|l|}{ ( $\leq 10$ años) } \\
\hline & $11-20$ años & 1,037743 & ,3918561 & 0,10 & 0,922 & ,495077 & 2,17524 \\
\hline & 21-38 años &, 5704459 & ,3745728 & $-0,85$ & 0,393 & ,1575029 & 2,066048 \\
\hline \multirow{3}{*}{ Ingresos } & \multicolumn{7}{|l|}{$(\geq 1.501 €)$} \\
\hline & $\leq 1.000 €$ & 6,944374 & 3,052124 & 4,41 & 0,000 & 2,934413 & 16,43406 \\
\hline & $1.001-1.500 €$ & 5,603139 & 1,90413 & 5,07 & 0,000 & 2,878494 & 10,9068 \\
\hline \multirow{2}{*}{ Tipo de contrato } & \multicolumn{7}{|l|}{ (Contrato indefinido) } \\
\hline & Duración determinada & ,9872973 & ,2881934 & $-0,04$ & 0,965 &, 5571615 & 1,749504 \\
\hline \multirow{2}{*}{ Jornada } & \multicolumn{7}{|l|}{ (Jornada completa) } \\
\hline & Jornada parcial & 1,701586 & ,7171859 & 1,26 & 0,207 & ,7448845 & 3,88704 \\
\hline \multirow{2}{*}{$\begin{array}{l}\text { Sector de } \\
\text { población }\end{array}$} & \multicolumn{7}{|l|}{ (Sectores población) } \\
\hline & Población en general &, 0813183 &, 050478 & $-4,04$ & 0,000 & ,0240884 & ,2745162 \\
\hline
\end{tabular}

\begin{tabular}{l|l}
\hline Number of obs & 387 \\
\hline LR chi' ${ }^{2}(19)$ & 103,57 \\
\hline Prob $>$ chi $^{2}$ & 0,0000 \\
\hline Pseudo $\boldsymbol{R}^{2}$ & 0,2270 \\
\hline Log likelihood & $-176,3896$ \\
\hline
\end{tabular}

*El valor de referencia se señala entre paréntesis.

Fuente: Elaboración propia. 
Entre todas las variables, se han incluido estas siete porque son las que están correlacionadas con trabajar en una entidad de la esfera relacional.

Como puede apreciarse, las variables estadísticamente significativas son convivencia, ingresos y sector de población. Cuando el profesional encuestado convive con sus padres, amigos o parientes, aumenta 1,90 veces la razón de trabajar en una entidad la esfera relacional. Tener ingresos de hasta 1.000 euros y de 1.001 a 1.500 euros incrementa la razón de trabajar en una entidad de la esfera relacional 6,94 veces y 5,60 veces, respectivamente. Cuando el entrevistado, desarrolla su actividad con la población en general, la razón de trabajar en una entidad de la esfera relacional disminuye 0,08 veces.

Finalmente, se ha de señalar que la variable que tiene más efecto sobre la probabilidad de trabajar en una entidad de la esfera estatal es el sector de población (población en general). El valor de 0,08 de variación asociado a la variable sector de población es equivalente a un efecto positivo de 12,5 $(1 / 0,08=12,5)$. Por tanto, el efecto de la variable sector de población es superior al efecto de la variable ingresos y convivencia.

\section{Conclusiones}

A lo largo de las páginas anteriores, hemos podido ver la influencia que ejercen en las diferentes esferas de la estructura social un conjunto de variables que hemos considerado fundamentales: sexo, edad, estado civil, convivencia, antigüedad en la profesión, salario, jornada, modalidad de contrato y sector de población.

Las conclusiones de este análisis, que han ido ya saliendo en los diferentes apartados, habrá que con- siderarlas en su valor exacto, pues, como sabemos, la muestra obtenida al hacer la encuesta, aunque suficiente, no ha sido de probabilidad, de forma que no somos capaces de decir los márgenes de error y la probabilidad de nuestras afirmaciones. Por todo ello, vamos a dejar nuestras conclusiones como tendencias observadas en este estudio:

- Las respuestas que los trabajadores sociales encuestados han dado en esta investigación han puesto de manifiesto que casi el $80 \%$ de los trabajadores sociales encuestados se encuentran ejerciendo la profesión, de los cuales el 58,4\% desarrollan su actividad profesional en la esfera estatal, el $29,9 \%$ en la esfera relacional y el $11,7 \%$ restante en la esfera mercantil.

- Las variables que tienen un efecto positivo sobre la probabilidad de trabajar en la esfera estatal son: edad (más probabilidad cuantos más años) y sector de población (población en general). Las variables convivencia e ingresos afectan negativamente (Figura 1).

- La variable que tiene un efecto positivo sobre la probabilidad de trabajar en una entidad de la esfera mercantil es ingresos (más probabilidad cuantos menos ingresos). La variable sector de población afecta negativamente (Figura 1).

- Las variables ingresos (más probabilidad cuantos menos ingresos) y convivir con sus padres, amigos o parientes tienen un efecto positivo sobre la probabilidad de trabajar en la esfera relacional. El sector población (población en general) afecta negativamente (Figura 1).

La carencia de una información más amplia y compleja sobre este tema ha limitado los resultados de nuestra investigación -cuyo objeto central no era éste, obviamente- a un repaso muy general de variables.

Figura 1. Probabilidad de que un profesional desarrolle su activad laboral en las diferentes esferas de la estructura social, en función de una serie de variables individuales y del puesto de trabajo

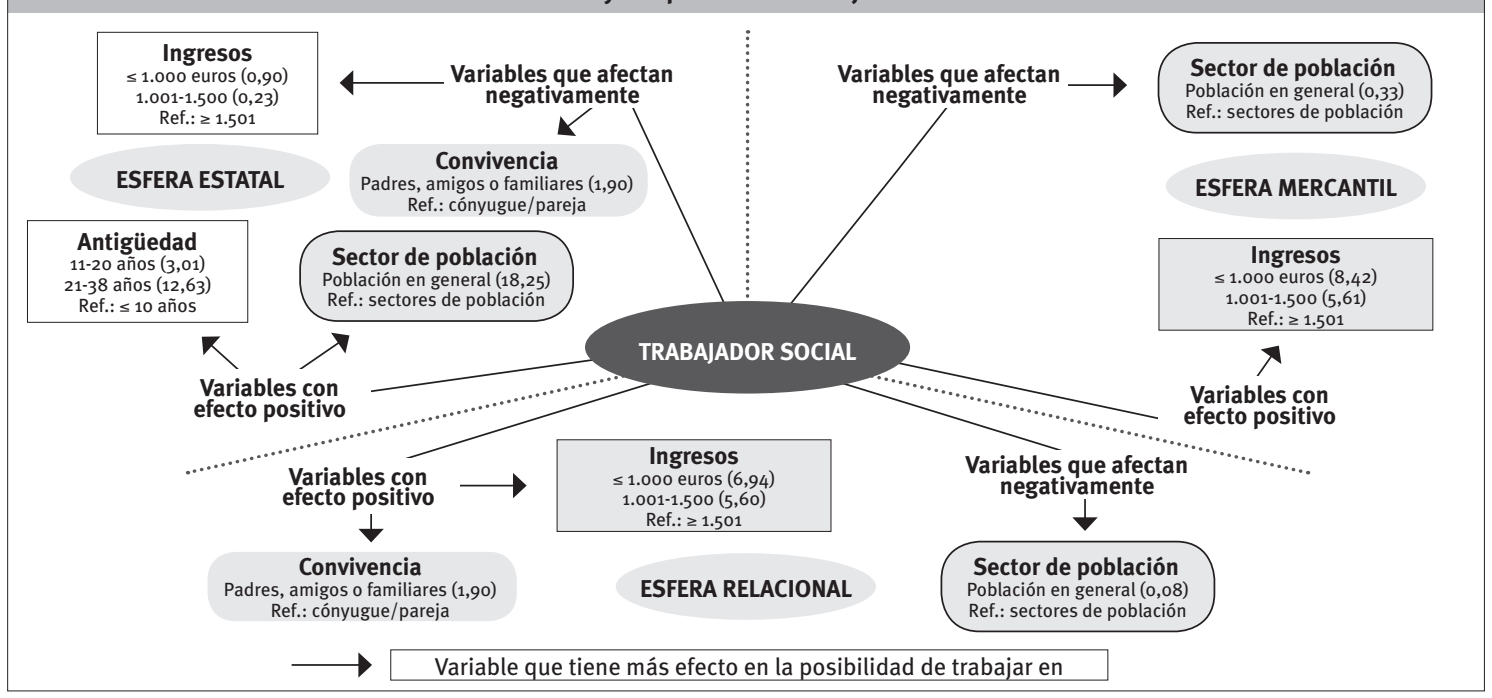

Nota: La razón, señalada entre paréntesis, representa la frecuencia de un suceso sobre la frecuencia de su no concurrencia. Fuente: Elaboración propia. 
ADELANTADO, J.; NOGUERA, J. A.; y RAMBLA, X. (2000): “El marco de análisis: las relaciones complejas entre estructura social y políticas sociales", en ADELANTADO, J. (coord.), Cambios en el Estado de bienestar. Políticas sociales y desigualdades en España, Barcelona, Icaria, págs. 23-61.

BANDA, T. et al. (1999): "Investigación sobre la ocupación profesional de los trabajadores sociales", Cuadernos de Trabajo Social, $\mathrm{n}$ - 12 , págs. 107-136.

BARBANO, F. (1969): “Significado y análisis de las estructuras en antropología y en sociología", en BARBANO, F. et al., Estructuralismo y sociología, Buenos Aires, Nueva Visión.

BERASALUZE, A.; y BERRIO-OTXOA, K. (2008): El ejercicio profesional del trabajo social hoy, VitoriaGasteiz, Colegios Oficiales de Diplomados/as en Trabajo Social de Araba, Bizkaia y Gipuzkoa.

CONSEJO DE EUROPA (2001): Recomendación REC (2001)1 del Comité de Ministros a los países miembros sobre los Trabajadores Sociales, adoptada por el Comité de Ministros del Consejo de Europa el 17 de enero de 2001 en su 737 reunión, Estrasburgo, Consejo de Europa.

CONSEJO GENERAL DEL TRABAJO SOCIAL (2009): Memoria 2009, Madrid, Consejo General del Trabajo Social.

CUESTA RUIZ, A. B. (coord.) (2007): Situación laboral de las trabajadoras sociales en La Rioja. Nuevos retos y propuestas de futuro, Logroño, Colegio Oficial de Diplomados en Trabajo Social y Asistentes Sociales de La Rioja.

FERNÁNDEZ, B. (coord.) (2002): Estudio sobre las trabajadoras sociales que ejercen su profesión en Navarra, Pamplona, UPNA.
FERNÁNDEZ GARCÍA, T.; y PONCE DE LEÓN, L. (2009): "Contribuciones de las ciencias sociales al trabajo social”, en FERNÁNDEZ, T. (coord.), Fundamentos del trabajo social, Madrid, Alianza Editorial, págs. 221-248.

GÓMEZ GARCÍA, R. (2010): El trabajo social en Castilla y León: estudio sociológico de una profesión, León, Universidad de León, Área de Publicaciones.

INSTITUTO ÁGORA (2001): Aproximación de la realidad profesional y formativa de los trabajadores sociales, Madrid, Consejo General de Colegios Oficiales de Diplomados en Trabajo Social y Asistentes Sociales

IZQUIETA, J. L.; CALLEJO, J. J.; y PRIETO, J. M. (2008): “EI tercer sector y las administraciones públicas. Relaciones en el ámbito de la atención social en los niveles regional y local", Revista Internacional de Sociología, vol. LXVI, nำ49, enero-abril, págs. 115-139.

KHAN, A J.; y KAMMERMAN, S. O. (1987): Los servicios sociales desde una perspectiva internacional. El sexto sistema de protección social, Madrid, Siglo XXI de España.

LEACH, E. R.; y UDY, S. H. Jr. (1974): “Estructura social. I. Historia del concepto", en SILLS, D. L. (dir.) Enciclopedia internacional de las ciencias sociales, vol. 4, Madrid, Aguilar, págs. 592-597.

LEVY, M. J. (1952): The Structure of Society, Princeton, Princeton University Press.

LLOVET, J. J.; y USIETO, R. (1990): Los trabajadores sociales. De la crisis de identidad a la profesionalización, Madrid, Popular.

MERTON, R. K. (1970): Teoría y estructura sociales, México D. F., Fondo de Cultura Económica. 
MILLS, C. W.; y GERTH, H. (1953): Character and Social Structure, Nueva York, Routledge and Kegan Paul.

MONZÓN, J. L. et al. (2004): El tercer sector no lucrativo en el Mediterráneo. La economía social de no mercado. I. España, Francia, Grecia, Italia y Portugal, Valencia, Ciriec-España.

PARDAVILA, B.; y ESNAL, M. (1985): "Unos datos sociolaborales básicos de los trabajadores sociales-asistentes sociales", Revista Servicios Sociales y Política Social, $\mathrm{n}$ ㅇ 3, págs. 74-85.
PARSONS, T. (1967): Ensayos de teoría sociológica, Buenos Aires, Paidós.

- (1966): El sistema social, Madrid, Editorial de la Revista de Occidente.

TORICES, A. (2010): Situación del trabajo social en España [tesina inédita], León, Universidad de León, Escuela Universitaria de Trabajo Social 'Ntra. Sra. del Camino'.

VÁZQUEZ, J. M. (dir.) (1971): Situación del Servicio Social en España, Madrid, Instituto de Sociología Aplicada. 